\title{
MORFEMAS DERIVATIVOS Y FLEXIVOS EN LA FORMACIÓN TOPONÍMICA DE LA PROVINCIA DE GUANACASTE, COSTA RICA
}

\author{
Flor Garita Hernández
}

\begin{abstract}
RESUMEN
En este estudio se hace un análisis de los morfemas derivativos y flexivos que entran en la formación de nombres comunes que, al usarse como nombres propios de accidentes geográficos o poblados, se convierten en topónimos. Los morfemas derivativos son muy variados y los abundanciales constituyen mayoría, seguidos de los diminutivos y despectivos. También hay diversidad de alomorfos para cada morfema. Los morfemas flexivos corresponden a las marcas de género y número. En el caso del género, hay bastante irregularidad morfemática mientras que el número es más irregular.
\end{abstract}

\begin{abstract}
This is an analysis of derivational and inflexional morphemes included in the formation of common nouns that, when used as proper nouns to designate geographical features or towns, become toponyms. Derivational morphemes are varied and abundance markers constitute the majority, followed by diminutives and pejorative markers. There is also a variety of allomorphs for each morpheme. Inflexional morphemes constitute the markers of gender and number. Gender, but not so in the case of number, shows high morphemic regularity.
\end{abstract}

\section{Introducción}

Los topónimos son los nombres geográficos en su más amplio sentido: los orónimos, nombres de montañas, cerros, lomas, puntas, picos, altos, bajos, filas, cordilleras y llanos; los hidrónimos comprenden las lagunas, lagos, ríos, esteros, quebradas, playas, bahías, islas, islotes, golfos y caños; los ecónimos, referidos específicamente a poblados, fincas y haciendas.

En el presente trabajo se hace un análisis de los morfemas derivativos y flexivos que entran en la formación de nombres comunes que, al usarse como nombres propios de elementos geográficos, se convierten en topónimos. 
Para este estudio se parte de los conceptos de morfología, morfema y alomorfo, desde el punto de vista de la lingüística moderna.

Matthews (1980) define la morfología como aquella rama de la lingüística que se interesa por las formas de las palabras en construcciones y usos diversos. Por su parte, la Real Academia dice que la morfología abstrae las palabras de su contexto y estudia las diferentes formas que pueden adquirir para representar las categorías gramaticales y establecer, además, los medios que el idioma emplea para enriquecer su léxico formando nuevas palabras con base en las ya existentes.

En resumen, la morfología estudia la forma de las palabras o categorías gramaticales (sustantivos, adjetivos, verbos, etc.). Las palabras están constituidas por morfemas. El morfema, a su vez, es la mínima forma, la más pequeña sucesión de fonemas dotada de significación. También, dice la Real Academia, se le llama formante. Así, el morfema es la unidad mínima del plano de la expresión que está en relación con el plano del significado. El morfema puede coincidir con una palabra, por ej. en sol, mar, en cuyo caso se habla de raíces libres; otras veces, el morfema es parte de una palabra o bien, introduce modificaciones al significado de esa palabra.

Pike y Halliday, citados por Mathews, definen el morfema como la unidad mínima, indivisible y primitiva. De acuerdo con estos teóricos todo morfema tiene varias características basadas en su aparición con otros morfemas, es decir, relaciones distribucionales: dónde aparecen en la palabra. Si los morfemas pueden pronunciarse solos, entre pausas, se les denomina formas separadas o libres; por el contrario, si nunca aparecen en aislamiento se llaman formas inseparables o atadas.

Otro criterio de clasificación es la distribución tradicional entre raíces y afijos. Las raíces son morfemas que constituyen clases abiertas y son muy numerosas. Los afijos son más limitados. Las raíces y los afijos se pueden distinguir de acuerdo con dos criterios: por el grado de abstracción del significado y por la capacidad que tienen para constituir por sí solos palabras. Por el grado de abstracción del significado, las raíces son morfemas con significados muy concretos en la realidad extralinguística, mientras que los afijos tienen significados muy abstractos e incluso, algunas veces, sin referentes en la realidad extralinguística. Son meros requisitos en la estructura de una lengua. Los afijos se clasifican según su colocación y según su función. Por su colocación, los afijos pueden ser prefijos, si van antes de la raíz, sufijos si van después e infijos si se insertan dentro de la raíz. Por su función, los afijos pueden ser derivativos o flexivos.

Los morfemas derivativos son los que modifican el significado de la raíz y sirven para construir temas. Los flexivos se unen al tema para indicar relaciones con otras palabras. Los morfemas derivativos constituyen clases arbitrarias y pueden cambiar la categoría de la palabra. El morfema flexivo no cambia la categoría de la palabra.

Por otra parte, los morfemas pueden tener más de una forma. Cuando el morfema tiene más de una forma para expresar el mismo fenómeno, se dice que estas otras formas son alomorfos del mismo morfema. Así, dos o más alomorfos pertenecen a un morfema si tienen el mismo significado y están en distribución complementaria, es decir, donde aparece uno, no aparece el otro. 


\section{Clasificación de los topónimos según el morfema que presentan}

\subsection{Topónimos con morfemas derivativos tipo sufijos}

\subsubsection{Morfemas con significación abundancial o colectiva}

\begin{tabular}{|c|c|c|c|}
\hline TOPÓNIMO & CARACTERÍSTICA & RAÍZ & SUFIJO \\
\hline Abejonal & Loma & abejon- & $-\mathrm{al}$ \\
\hline Achiotal & Estero / Poblado & achiot- & $-\mathrm{al}$ \\
\hline Aguabal & Cerros / Río & $\operatorname{agu}(a)(b)$ & -al \\
\hline Arenal & Río & aren- & $-a l$ \\
\hline Aromal & Finca & arom- & $-a l$ \\
\hline Arrozal & Quebrada / Loma & arroz- & -al \\
\hline Azufrales & Quebrada & azufr- & $-\mathrm{al}(\mathrm{es})$ \\
\hline Ayotal & Quebrada & ayot- & $-\mathrm{al}$ \\
\hline Balsar & Quebrada & bals- & -ar \\
\hline Balsal & Cerro / Poblado & bals- & $-\mathrm{al}$ \\
\hline Barbudal & Quebrada / Pobl. / Cerro & barbud- & - al \\
\hline Barrial & Quebrada & barri- & $-a l$ \\
\hline Bijagual & Poblado & bijagu- & -al \\
\hline Brasilar & Estero / Cerro & brasil- & -ar \\
\hline Cabuyal & Estero / Punta & cabuy- & -al \\
\hline Caimital & Quebrada / Poblado & caimit- & -al \\
\hline Camaronal & Playa / Punta / Hacienda & camaron- & -al \\
\hline Cañablancal & Laguna & cañablanc- & -al \\
\hline Cañal & Quebrada / Poblado & cañ- & -al \\
\hline Canelar & Quebrada & canel- & -ar \\
\hline Cangrejal & Quebrada / Poblado & cangrej- & $-a l$ \\
\hline Carbonal & Quebrada & carbon- & $-\mathrm{al}$ \\
\hline Capulinal & Loma & capulin- & $-a l$ \\
\hline Carrizal & Playa / Poblado & carriz- & -al \\
\hline Cascajal & Quebrada / Cerro & cascaj- & -al \\
\hline Cenizarada & Estero & cenizar- & -ada \\
\hline Castillal & Quebrada & castill- & -al \\
\hline Chanal & Finca & chan- & -al \\
\hline Chapernal & Quebrada / Finca & chapern- & $-a l$ \\
\hline Colmenar & Poblado & colmen- & -ar \\
\hline Cocal & Playa & coc- & -al \\
\hline Colegallal & Cerro & colegall- & -al \\
\hline Conchal & Playa / Poblado / Cerro & conch- & $-a l$ \\
\hline Congal & Poblado & cong- & $-a l$ \\
\hline Correntadas & Poblado & corrent- & -ada -s \\
\hline Cortezal & Finca & cortez- & $-a l$ \\
\hline
\end{tabular}




\begin{tabular}{|c|c|c|c|}
\hline Coyolar & Quebrada / Poblado / Cerro & coyol- & -ar \\
\hline Espavelar & Quebrada / Finca & espavel- & -ar \\
\hline Frijolar & Playa / Punta & frijol- & -ar \\
\hline Corozalito & Poblado & coroz- & -al (it) -o \\
\hline Gamalotal & Río & gamalot- & -al \\
\hline Corozal & Poblado & coroz- & -al \\
\hline Guaitilar & Cerro & guait- & -ar \\
\hline Guastomatal & Quebrada / Poblado & guast- & $-\mathrm{al}$ \\
\hline Guayabal & Laguna / Poblado & guayab- & $-a l$ \\
\hline Hachal & Cerro & hach- & $-a l$ \\
\hline Higueronal & Loma & higueron- & $-a l$ \\
\hline Icacal & Estero & icac- & -al \\
\hline Jazminal & Quebrada / Poblado / Fila & jazmin- & -al \\
\hline Junquillal & Bahía / Poblado & junquill- & -al \\
\hline Laurelal & Cerro & laurel- & -al \\
\hline Limonal & Quebrada / Poblado / Cerro & limon- & -al \\
\hline Maderal & Quebrada / Finca & mader- & -al \\
\hline Madroñal & Loma & madroñ- & -al \\
\hline Maicillal & Río / Poblado / Fila & maicill- & -al \\
\hline Manzanales & Cerro & manzan- & -al -es \\
\hline Maquencal & Quebrada / Poblado / Cerro & maquenc- & -al \\
\hline Manglar & Poblado & mangl- & -ar \\
\hline Millal & Quebrada / Poblado & mill- & -al \\
\hline Mojal & Quebrada / Hacienda & moj- & -al \\
\hline Moral & Laguna & mor- & $-a l$ \\
\hline Metalada & Laguna & metal- & -ada \\
\hline Mostrencal & Playa & mostrenc- & $-a l$ \\
\hline Mozotal & Quebrada / Poblado / Cerro & mozot- & -al \\
\hline Nancital & Quebrada / Poblado / Cerro & nancit- & $-a l$ \\
\hline Nambiral & Fila & nambir- & - al \\
\hline Naranjal & Quebrada / Poblado & naranj- & $-a l$ \\
\hline Nisperal & Quebrada / Punta & nisper- & $-a l$ \\
\hline Ocotal & Bahía / Cerro & ocot- & - al \\
\hline Ojochada & Laguna & ojoch- & -ada \\
\hline Ojochal & Poblado / Llanos & ojoch- & -al \\
\hline Ojochillal & Quebrada / Cerro & ojochill- & -al \\
\hline Ostional & Estero / Poblado / Punta & ostion- & -al \\
\hline Pacayal & Quebrada & pacay- & -al \\
\hline Palmar & Quebrada / Poblado / Cerro & palm- & -ar \\
\hline Palmichal & Quebrada & palmich- & $-a l$ \\
\hline Papatular & Estero & papatul- & $-a r$ \\
\hline Pedernal & Río / Poblado / Cerro & pedern- & -al \\
\hline Pedregal & Quebrada / Poblado / Cerro & pedreg- & $-a l$ \\
\hline Pencal & Playa / Poblado & penc- & $-a l$ \\
\hline
\end{tabular}




$\begin{array}{llll}\text { Pirital } & \text { Cerro } & \text { pirit- } & \text {-al } \\ \text { Pital } & \text { Quebrada / Poblado } & \text { pit- } & \text {-al } \\ \text { Platanar } & \text { Estero / Finca } & \text { plat- } & \text {-ar } \\ \text { Polvazal } & \text { Poblado } & \text { polvaz- } & \text {-al } \\ \text { Polvazales } & \text { Cerro / Poblado } & \text { polvaz- } & \text {-al -es } \\ \text { Porozal } & \text { Poblado } & \text { poroz- } & \text {-al } \\ \text { Raizal } & \text { Quebrada / Finca } & \text { raiz- } & \text {-al } \\ \text { Pochotada } & \text { Lomas } & \text { pochot- } & \text {-ada } \\ \text { Roblar } & \text { Estero / Poblado } & \text { robl- } & \text {-ar } \\ \text { Ramadas } & \text { Quebrada / Río } & \text { ram- } & \text {-ada -s } \\ \text { Salitral } & \text { Quebrada / Poblado } & \text { salitr- } & \text {-al } \\ \text { Sandillal } & \text { Río } & \text { sandill- } & \text {-al } \\ \text { Sardinal } & \text { Río / Poblado } & \text { sardin- } & \text {-al } \\ \text { Sonzapotal } & \text { Quebrada } & \text { sonzapot- } & \text {-al } \\ \text { Tempatal } & \text { Poblado } & \text { tempat- } & \text {-al } \\ \text { Tortugal } & \text { Laguna / Poblado } & \text { tortug- } & \text {-al } \\ \text { Trigal } & \text { Quebrada } & \text { trig- } & \text {-al } \\ \text { Trompillal } & \text { Poblado } & \text { trompill- } & \text {-al } \\ \text { Yucal } & \text { Loma } & \text { yuc- } & \text {-al } \\ \text { Zapotal } & \text { Río / Poblado } & \text { zapot- } & \text {-al } \\ \text { Zapotillal } & \text { Bahía } & \text { zapotill- } & \text {-al } \\ \text { Zapal } & \text { Finca } & \text { zap- } & \text {-al } \\ \text { Ranchería } & \text { Quebrada } & \text { ranch(er)- } & \text {-í -a } \\ \text { Turrujal } & \text { Estero } & \text { turruj- } & \text {-al } \\ \text { Palizada } & \text { Cerro } & \text { pal(iz)- } & \text {-ada } \\ \text { Papaturrada } & \text { Estero } & \text { papaturr- } & \text {-ada } \\ & & & \end{array}$

\subsubsection{Morfemas con significado diminutivo}

TOPÓNIMO CARACTERÍSTICA

Alajuelita

Aguacatico

Arbolito

Bejuquito

Brasilito

Cabrita

Caimitalito

Canjelito

Cañita

Casitas

Cebollín

Ceibitas

Chiquita
Poblado

Quebrada

Poblado / Playa / Quebrada

Quebrada

Poblado/Playa/Bahía/Quebrada/Río brasil-

Quebrada

Poblado

Poblado/Estero/Río

Quebrada / Río

Poblado

Quebrada

Finca

Laguna

ceboll-
RAÍZ

alajuel-

aguacat-

arbol-

bejuqu-

cabr-

caimit(al)

canjel-

cañ-

cas-

ceib-

chiqu-
SUFIJOS

dim. gén. núm.

-it -a

-ic $-\mathrm{O}$

-it $\quad-0$

-it $-\mathrm{O}$

-it $\quad-0$

-it $\quad-a$

-it $\quad-0$

-it $-\mathrm{o}$

-it $\quad-$ a

-it $\quad-a \quad-s$

-ín

-it $\quad-a \quad-s$

-it -a 


\begin{tabular}{|c|c|c|c|c|}
\hline Chiquito & Quebrada / Río / Cerro & chiqu- & -it & -0 \\
\hline Corocito & Finca & coroc- & -it & $-\mathrm{O}$ \\
\hline Concavitas & Islote & concav- & -it & $-\mathrm{a}$ \\
\hline Coquito & Bahía & coqu- & - it & -0 \\
\hline Corocitos & Poblado / Llanos & coroc- & - it & -0 \\
\hline Corozalito & Poblado / Playa / Río & $\operatorname{coroz}(\mathrm{al})-$ & -it & -0 \\
\hline Coyolito & Poblado / Río / Alto & coyol- & -it & -0 \\
\hline Coyolarcitos & Quebrada & coyol(ar)- & - cit & -0 \\
\hline Florecita & Finca / Quebrada & flor- & -ecit & $-\mathrm{a}$ \\
\hline Granjita & Quebrada & granj- & -it & $-\mathrm{a}$ \\
\hline Guacalito & Finca / Quebrada & guacal- & -it & -0 \\
\hline Guacamayita & Playa & guacamay- & -it & $-\mathrm{a}$ \\
\hline Huacalito & Finca / Quebrada / Cerro & huacal- & -it & $-\mathrm{O}$ \\
\hline Huaquitas & Poblado & huaqu- & - it & $-\mathrm{a}$ \\
\hline Huaquitos & Lomas & huaqu- & - it & -0 \\
\hline Iguanita & Poblado / Bahía / Estero / Playa & iguan- & -it & $-\mathrm{a}$ \\
\hline Islita & Poblado / Río / Quebrada / Punta & isl- & -it & $-\mathrm{a}$ \\
\hline Jicarito & Estero / Quebrada & jicar- & - it & $-\mathrm{o}$ \\
\hline Jobitos & Laguna / Loma & job- & - it & $-\mathrm{o}$ \\
\hline Lucecita & Finca & luc- & -ecit & $-\mathrm{a}$ \\
\hline Lajitas & Quebrada & laj- & - it & $-a$ \\
\hline Lomitas & Poblado & lom- & -it & $-\mathrm{a}$ \\
\hline Lloroncita & Quebrada & llor(on)- & - cit & $-\mathrm{a}$ \\
\hline Moralito & Finca & moral- & -it & -0 \\
\hline Matapalito & Bahía / Playa / Punta & matapal- & -it & $-\mathrm{O}$ \\
\hline Naranjalito & Poblado & naranj(al)- & -it & $-\mathrm{O}$ \\
\hline Montañita & Quebrada & montañ- & -it & $-\mathrm{a}$ \\
\hline Naranjitos(Agrios) & Poblado & naranj- & -it & -0 \\
\hline Moralito & Estero & mor(al)- & -it & -0 \\
\hline Nosarita & Poblado & nosar- & - it & $-a$ \\
\hline Morritos & Playa / Punta & morr- & -it & $-\mathrm{O}$ \\
\hline Palmita & Poblado / Cerro & palm- & -it & $-\mathrm{a}$ \\
\hline Mosquito & Quebrada / Islas / Laguna & mosq- & -it & -0 \\
\hline Ranchitos & Poblado & ranch- & -it & -0 \\
\hline Nacascolito & Playa & nacascol- & -it & -0 \\
\hline Sabalito & Poblado / Quebrada & sabal- & -it & -0 \\
\hline Negritos & Quebrada & negr- & -it & -0 \\
\hline Salinitas & Poblado & sal(in)- & -it & $-\mathrm{a}$ \\
\hline Papelito & Quebrada & papel- & - it & -0 \\
\hline Talolinguita & Poblado & talolingu- & -it & $-a$ \\
\hline Pocitas & Quebrada & poc- & - it & $-\mathrm{a}$ \\
\hline Pocito & Quebrada & poc- & -it & -0 \\
\hline Pocitos & Río & poc- & -it & -0 \\
\hline Uvita & Poblado & uv- & - it & $-a$ \\
\hline
\end{tabular}




\begin{tabular}{|c|c|c|c|c|}
\hline Yegüitas & Quebrada & yegü- & -it & $-\mathrm{a}$ \\
\hline Zanjita & Poblado & zanj- & -it & $-a$ \\
\hline Playitas & Playas / Laguna & play- & -it & $-a$ \\
\hline Pilitas & Quebrada & pil- & - it & $-\mathrm{a}$ \\
\hline Pajarito & Laguna & pajar- & -it & -0 \\
\hline Ramita & Laguna & ram- & -it & $-\mathrm{a}$ \\
\hline Saltito & Quebrada & salt- & -it & -0 \\
\hline Sequito & Río & sequ- & - it & -0 \\
\hline Tempisquito & Río & tempisqu- & -it & -0 \\
\hline Tronadorcita & Quebrada & $\operatorname{tron}(\mathrm{a})(\mathrm{dor})-$ & - cit & $-\mathrm{a}$ \\
\hline Caballito & Cerro & caball- & -it & -0 \\
\hline Cerrito & Cerro & cerr- & -it & -0 \\
\hline Cerritos & Punta & cerr- & -it & -0 \\
\hline Cebollín & Cerro / Lomas & ceboll- & -ín & \\
\hline Orosilito & Cerro & oros(il)- & -it & -0 \\
\hline Molino & Quebrada & mol- & -in & -0 \\
\hline Clavelinas & Río & clavel- & -in & $-\mathrm{a}$ \\
\hline Piñuela & Quebrada / Cerro & piñ- & -uel & $-a$ \\
\hline Plazuela & Poblado / Quebrada & plaz- & -uel & $-\mathrm{a}$ \\
\hline Cornizuelo & Laguna & corn(iz)- & -uel & -0 \\
\hline Cajeta & Quebrada & caj- & -et & $-\mathrm{a}$ \\
\hline Calceta & Quebrada & calc- & -et & $-a$ \\
\hline Caleta & Quebrada & cal- & -et & $-\mathrm{a}$ \\
\hline Isleta & Loma / Poblado & isl- & -et & $-a$ \\
\hline Pileta & Quebrada & pil- & -et & $-\mathrm{a}$ \\
\hline Piletas & Quebrada & pil- & -et & $-\mathrm{a}$ \\
\hline Boquete & Cerro & boqu- & -et & $-e$ \\
\hline Carreta & Cerro & carr- & -et & $-a$ \\
\hline
\end{tabular}

\subsubsection{Morfemas con significado despectivo}

\section{TOPÓNIMO CARACTERÍSTICA}

Asientillo

Bolillos

Arenilla

Conventillos

Corralillo

Esterillas

Guanacastillo

Hatillo

Camastro

Higuerilla

Hornillas
Quebrada

Poblado / Cerro

Punta

Hacienda / Río

Poblado / Laguna / Loma

Quebrada

Quebrada

Hacienda / Quebrada

Loma

Poblado

Quebrada
RAÍZ

asient-

bol-

aren-

convent-

corral-

ester-

guanacast-

hat-

cam(a)-

higu(er)-

horn-
SUFIJO

-ill -o

$\begin{array}{lll}- \text { ill } & -0 & -s\end{array}$

-ill -a

$\begin{array}{lll}- \text { ill } & -\mathrm{O} & -\mathrm{s}\end{array}$

-ill $-\mathrm{O}$

-ill -a

-ill $-\mathrm{O}$

-ill $-\mathrm{O}$

-str -o

-ill $\quad-$ a

-ill $\quad-a \quad-s$ 


\begin{tabular}{|c|c|c|c|c|}
\hline Junquillal & Bahía & junqu- & -ill(al) & \\
\hline Lagartillo & Quebrada & lagart- & -ill & $-\mathrm{O}$ \\
\hline Platanillo & Poblado / Laguna / Río / Cerro & platan- & -ill & -o \\
\hline Leoncillo & Isla & leon- & - cill & $-\mathrm{O}$ \\
\hline Portillo & Finca / Cerro & port- & -ill & -o \\
\hline Potrerillos & Hacienda & potrer- & -ill & -o \\
\hline Manzanillo & Bahía / Playa / Quebrada / Punta & manzan- & -ill & -o \\
\hline Montecillo & Laguna & mont- & -ecill - & -o \\
\hline Maicillal & Fila & maic- & -ill(al) & \\
\hline Ojochillal & Quebrada / Cerro & ojoch- & -ill(al) & \\
\hline Zapatillo & Quebrada & zapat- & - ill & -o \\
\hline Zapotillal & Bahía / Playa & zapot- & - ill(al) & \\
\hline Coralillo & Cerro & coral- & -ill & $-\mathrm{o}$ \\
\hline Crucillas & Fila & cruc- & -ill & $-\mathrm{a}$ \\
\hline Jocotillo & Cerros & jocot- & -ill & $-\mathrm{O}$ \\
\hline Molinillo & Cerro & molin- & -ill & -0 \\
\hline Palizada & Cerro & pal- & -iz(ada) & \\
\hline Quebracho & Cerro / Loma & quebr(a)- & -cho & \\
\hline Sabanilla & Loma & saban- & -ill & $-\mathrm{a}$ \\
\hline Granadilla & Quebrada & granad- & - ill & $-\mathrm{a}$ \\
\hline Laguna & Finca & lag- & -un & $-\mathrm{a}$ \\
\hline
\end{tabular}

\subsubsection{Morfemas que expresan lugar, instalación o vinculación con algo}

\begin{tabular}{|c|c|c|c|c|}
\hline TOPÓNIMO & CARACTERÍSTICA & RAÍZ & \multicolumn{2}{|c|}{ SUFIJO } \\
\hline Aserradero & Quebrada & $\operatorname{aserr}(a)(d)-$ & -er & -0 \\
\hline Bebedero & Poblado & bebed- & -er & -0 \\
\hline Botadero & Cerro & $\operatorname{bot}(a)(d)$ & -er & -0 \\
\hline Calera & Quebrada / Río & cal- & -er & $-\mathrm{a}$ \\
\hline Carbonera & Finca / Cerro & carbon- & -er & $-\mathrm{a}$ \\
\hline Coyolera & Playa & coyol- & -er & $-\mathrm{a}$ \\
\hline Chiquero & Quebrada / Bajo & chiqu- & -er & -0 \\
\hline Choricera & Poblado & choric- & -er & $-\mathrm{a}$ \\
\hline Coyotera & Quebrada & coyot- & -er & $-a$ \\
\hline Cementerio & Playa & cement- & $-e r(i)$ & -0 \\
\hline Embarcadero & Poblado & embarc(a)(d) & -er & -0 \\
\hline Hormiguero & Quebrada / Cerro & hormig- & -er & -0 \\
\hline Lindero & Poblado / Quebrada & lind- & -er & -0 \\
\hline Lagartero & Estero / Cerro & lagart- & -er & -0 \\
\hline Ponedero & Poblado & poned- & -er & -0 \\
\hline Huesera & Quebrada & hues- & -er & $-\mathrm{a}$ \\
\hline Pradera & Finca & prad- & -er & $-a$ \\
\hline Moledero & Cerro & mol(ed)- & -er & -0 \\
\hline
\end{tabular}




$\begin{array}{ll}\text { Quesera } & \text { Poblado / Quebrada } \\ \text { Cabeceras } & \text { Quebrada } \\ \text { Despeñadero } & \text { Quebrada } \\ \text { Campero } & \text { Río } \\ \text { Divisadero } & \text { Quebrada / Cerro / Loma } \\ \text { Lajero } & \text { Quebrada } \\ \text { Resbaladero } & \text { Quebrada } \\ \text { Raspadero } & \text { Cerro } \\ \text { Sombrero } & \text { Playa / Cerro } \\ \text { Potrero } & \text { Poblado / Bajo / Cerro } \\ \text { Terrero } & \text { Quebrada } \\ \text { Terreros } & \text { Quebrada / Fila } \\ \text { Tiestero } & \text { Quebrada } \\ \text { Tranquera } & \text { Quebrada } \\ \text { Tucera } & \text { Quebrada } \\ \text { Boquerones } & \text { Loma }\end{array}$

\begin{tabular}{|c|c|c|}
\hline ques- & -er & $-\mathrm{a}$ \\
\hline cabec- & -er & $-\mathrm{a}$ \\
\hline (des)peñ(a)(d)- & -er & -0 \\
\hline camp- & -er & -0 \\
\hline $\operatorname{divis}(a)(d)-$ & -er & -0 \\
\hline laj- & -er & -0 \\
\hline $\operatorname{resbal}(a)(d)-$ & -er & -0 \\
\hline $\operatorname{rasp}(a)(d)-$ & -er & -0 \\
\hline sombr- & -er & -0 \\
\hline potr- & -er & -0 \\
\hline terr- & -er & -0 \\
\hline terr- & -er & -0 \\
\hline tiest- & -er & -0 \\
\hline tranqu- & -er & $-\mathrm{a}$ \\
\hline tuc- & -er & $-\mathrm{a}$ \\
\hline boqu- & -er(on) & -es \\
\hline
\end{tabular}

\subsubsection{Morfemas con significado aumentativo}

\begin{tabular}{|c|c|c|c|c|}
\hline TOPÓNIMO & CARACTERÍSTICA & RAÍZ & \multicolumn{2}{|c|}{ SUFIJO } \\
\hline Bolsón & Poblado / Cerro & bols- & -ón & \\
\hline Cajón & Poblado & caj- & -ón & \\
\hline Higuerón & Poblado / Quebrada / Río & higuer- & -ón & \\
\hline Callejones & Playa & call(ej)- & -ón & -es \\
\hline Llorona & Finca / Quebrada & llor- & -on & $-\mathrm{a}$ \\
\hline Troncón & Finca / Río & tronc- & -on & \\
\hline Dormilona & Quebrada & dormil- & -on & $-a$ \\
\hline Esterón & Quebrada & ester- & -ón & \\
\hline Esterones & Quebrada & ester- & -on & -es \\
\hline Garrapatón & Quebrada & garrapat- & -ón & \\
\hline Garzón & Río & garz- & -ón & \\
\hline Huertón & Laguna / Quebrada & huert- & -ón & \\
\hline Lajones & Quebrada & laj- & -on & -es \\
\hline Playones & Poblado & play- & -on & -es \\
\hline Pajarones & Quebrada & pajar- & -on & -es \\
\hline Peñasco & Quebrada & peñ- & -asc & -0 \\
\hline Picazón & Estero & pic(az)- & -ón & \\
\hline Pilonas & Quebrada & pil- & -on & $-\mathrm{a}$ \\
\hline Pozón & Quebrada / Cerros & poz- & -ón & \\
\hline Zanjones & Quebrada & zanj- & -on & -es \\
\hline Boquerones & Cerro / Loma & boqu(er) & -on & -es \\
\hline Charcones & Cerro & charc- & -on & -es \\
\hline Copetón & Cerro & copet- & -ón & \\
\hline Terrón & Cerro & err- & -ón & \\
\hline
\end{tabular}


2.1.6. Morfemas que expresan fundamentalmente idea de acción, efecto o acción-efecto

\begin{tabular}{|c|c|c|c|c|}
\hline TOPÓNIMO & CARACTERÍSTICA & RAÍZ & SUFIJO & \\
\hline Angostura & Poblado & angost- & -ur & $-a$ \\
\hline Calentura' & Quebrada & calent- & -ur & $-\mathrm{a}$ \\
\hline Desprendimiento & Quebrada & (des)prend(i)- & -mient & -0 \\
\hline Florida & Quebrada & flor- & $-\mathrm{id}$ & $-\mathrm{a}$ \\
\hline Henchidero & Río & henchi(d)- & -er & -0 \\
\hline Pelón & Quebrada / Cerro & pel- & -ón & \\
\hline Pelona & Quebrada / Cerro & pel- & -on & $-\mathrm{a}$ \\
\hline Pelonas & Islas & pel- & -on & $-a$ \\
\hline Pescadero & Río & $\operatorname{pesc}(a)(d)-$ & -er & -0 \\
\hline Pintura & Quebrada & pint- & -ur & $-\mathrm{a}$ \\
\hline Virador & Bahía & $\operatorname{vir}(a)-$ & -dor & \\
\hline Viradores & Islas & $\operatorname{vir}(\mathrm{a})-$ & -dor & -es \\
\hline Coronación & Cerro & $\operatorname{coron}(a)-$ & -ción & \\
\hline Mirador & Alto & $\operatorname{mir}(a)-$ & -dor & \\
\hline Gotera & Poblado & got- & -er & $-\mathrm{a}$ \\
\hline Contentera & Quebrada / Cerros & content- & -er & $-\mathrm{a}$ \\
\hline
\end{tabular}

2.1.7. Morfemas que expresan cualidades del topónimo

\begin{tabular}{|c|c|c|c|c|c|}
\hline TOPÓNIMO & CARACTERÍSTICA & RAÍZ & \multicolumn{3}{|c|}{ SUFIJO } \\
\hline Arenosa & Quebrada & aren- & - os & $-\mathrm{a}$ & \\
\hline Barrosa & Poblado & barr- & - os & $-\mathrm{a}$ & \\
\hline Calosa & Quebrada & cal- & $-\mathrm{os}$ & $-\mathrm{a}$ & \\
\hline Bulliciosa & Quebrada & bull(ici)- & - os & $-\mathrm{a}$ & \\
\hline Espavelosa & Laguna & espavel- & $-\mathrm{os}$ & $-\mathrm{a}$ & \\
\hline Gusanosa & Quebrada & gusan- & - os & $-\mathrm{a}$ & \\
\hline Lajosa & Quebrada & laj- & - os & $-\mathrm{a}$ & \\
\hline Montosa & Isla / Loma & mont- & -os & $-\mathrm{a}$ & \\
\hline Pedregosa & Finca / Cerros / Fila & pedreg- & - os & $-a$ & \\
\hline Salinas & Poblado / Estero / Punta & sal- & -in & $-\mathrm{a}$ & $-s$ \\
\hline Salinitas & Poblado & sal- & -in(it) & $-a$ & $-s$ \\
\hline Sainalosa & Laguna & sain(al)- & - os & $-\mathrm{a}$ & \\
\hline Salina & Río & sal- & -in & $-\mathrm{a}$ & \\
\hline Cenizosa & Fila & ceniz- & - os & $-\mathrm{a}$ & \\
\hline Felicidad & Finca & felic(i)- & $-\mathrm{dad}(\mathrm{s}$ & sentic & abstracto) \\
\hline Esperanza & Quebrada / Finca & esper- & $-a n z$ & $-\mathrm{a}$ & \\
\hline Barrialosa & Quebrada & barr(i)(al)- & -os & $-\mathrm{a}$ & \\
\hline Tollosa & Playa & toll- & -Os & $-\mathrm{a}$ & \\
\hline Toyoso & Río & toll- & -os & -0 & \\
\hline
\end{tabular}


2.1.8. Morfemas que expresan intensificación de la cualidad

\begin{tabular}{llllll} 
TOPÓNIMO & CARACTERÍSTICA & RAÍZ & & \multicolumn{2}{c}{ SUFIJO } \\
& & & & & \\
Brojudo & Cerro & broj- & -ud & $-o$ & \\
Picuda & Cerro / Loma & pic- & -ud & - a & \\
Picudas & Fila & pic- & -ud & $-\mathrm{a}$ & -s
\end{tabular}

2.1.9. Morfemas con significado de agente

$\begin{array}{llll}\text { TOPÓNIMO } & \text { CARACTERÍSTICA } & \text { RAÍZZ } & \text { SUFIJO } \\ \text { Chupador } & \text { Finca } & \text { chup-a- } & \text {-dor } \\ \text { Escarbador } & \text { Finca } & \text { escarb(a)- } & \text {-dor } \\ \text { Tronadora } & \text { Poblado / Río } & \text { tron(a)- } & \text {-dor -a } \\ \text { Ranchería } & \text { Quebrada } & \text { ranch- } & \text {-er(í) -a }\end{array}$

2.1.10. Morfemas con significado de gentilicio

\begin{tabular}{|c|c|c|c|}
\hline TOPÓNIMO & CARACTERÍSTICA & RAÍZ & SUFIJO \\
\hline Francesa & Finca & franc- & -es \\
\hline Española & Finca & españ- & $-\mathrm{ol}$ \\
\hline
\end{tabular}

2.1.11. Morfemas con significado de oficio

TOPÓNIMO CARACTERÍSTICA RAÍZ SUFIJO

Cocinero Isla cocin- $\quad$-er $\quad$ (o)

2.1.12. Morfemas con significado colectivo

TOPÓNIMO CARACTERÍSTICA RAÍZ SUFIJO

$\begin{array}{lllll}\text { Ranchería Quebrada } & \text { Ranch- } & \text { (-er) } & \text {-í } & \text {-a }\end{array}$

\subsection{Topónimos con morfemas derivativos tipo prefijos}

\section{TOPÓNIMO CARACTERÍSTICA PREFIJO RAÍZ}

$\begin{array}{llll}\text { Desechos } & \text { Quebrada } & \text { des- } & \text { ech(o)(s) } \\ \text { Desjarretado } & \text { Poblado } & \text { des- } & \text { jarret(a)(d)(o) } \\ \text { Desengaño } & \text { Quebrada } & \text { des- } & \text { engañ(o) } \\ \text { Despeñadero } & \text { Quebrada } & \text { des- } & \text { peñ(a)(d)(er)(o) }\end{array}$




$\begin{array}{llll}\text { Desprendimiento } & \text { Quebrada } & \text { des- } & \text { prend(i)(miento) } \\ \text { Embarcadero } & \text { Poblado } & \text { em- } & \text { barc(a)(d)(er)(o) } \\ \text { Enmedio } & \text { Río } & \text { en- } & \text { medi(o) } \\ \text { Enramada } & \text { Quebrada } & \text { en- } & \text { ram(a)(d)(a) } \\ \text { Reforma } & \text { Finca } & \text { re- } & \text { form(a) } \\ \text { Refundores } & \text { Finca } & \text { re- } & \text { fund(or)(es) } \\ \text { Rejoya } & \text { Finca / Cerros } & \text { re- } & \text { joy(a) } \\ \text { Abajo } & \text { Punta } & \text { a- } & \text { baj(o) } \\ \text { Atravesado } & \text { Cerro } & \text { a- } & \text { traves(a)(d)(o) } \\ \text { Rempujo } & \text { Loma } & \text { re-em- } & \text { puj-(o) }\end{array}$

\subsection{Topónimos con morfemas flexivos}

En esta parte se presentan las raíces libres o atadas que toman los morfemas de género femenino o masculino y de número plural; raíces atadas con morfemas derivativos a los cuales se les agregan los morfemas de femenino, masculino o plural y raíces atadas formadoras de participios - adjetivos, que, por su condición de derivados verbales, presentan los morfemas típicos de esta forma no personal.

2.3.1. Raíces libres o atadas con morfemas de género masculino o femenino y de número plural o de ambos

$\begin{array}{llll}\text { Abajo } & \text { Alcornoque } & \text { Almendro } & \text { Alta } \\ \text { Alto } & \text { Aduana } & \text { Agria } & \text { Agua } \\ \text { Amores } & \text { Almuerzos } & \text { Abandonos } & \text { Agujas } \\ \text { Aguacate } & \text { Amapola } & \text { Aguacata } & \text { Almendros } \\ \text { Amo } & \text { Angeles } & \text { Animas } & \text { Almuerzo } \\ \text { Anona } & \text { Anonos } & \text { Arcos } & \text { Aprecio } \\ \text { Arado } & \text { Arena } & \text { Ariete } & \text { Armado } \\ \text { Aromos } & \text { Avellanas } & \text { Arrayanes } & \text { Ayote } \\ \text { Ayotes } & \text { Azufrales } & \text { Bagre } & \text { Ballena } \\ \text { Bambú } & \text { Bejuco } & \text { Berros } & \\ \text { Bijagua } & \text { Blanca } & \text { Blanco } & \text { Bocana } \\ \text { Bochinche } & \text { Bolsa } & \text { Bongo } & \text { Bonita } \\ \text { Botas } & \text { Bote } & \text { Botija } & \text { Brisas } \\ \text { Bruja } & \text { Brujo } & \text { Burras } & \text { Burro } \\ \text { Buena } & \text { Caballo } & \text { Caballos } & \text { Cabra } \\ \text { Cabros } & \text { Cahuya } & \text { Cahuyo } & \text { Cacao } \\ \text { Cachimbo } & \text { Cacho } & \text { Calavera } & \text { Cales } \\ \text { Callo } & \text { Cambio } & \text { Camones } & \text { Camarones } \\ \text { Campero } & \text { Canales } & \text { Canelo } & \text { Caimito } \\ \text { Caña } & \text { Caja } & \text { Camas } & \text { Carao } \\ \text { Caoba } & \text { Carana } & \text { Carne } & \text { Carpa } \\ \text { Casa } & \text { Catarata } & \text { Cedro } & \text { Cedros } \\ & & & \end{array}$




\begin{tabular}{|c|c|c|c|}
\hline Ceiba & Cepo & Cenízaro & Centinela \\
\hline Chancha & Chancho & Chanchos & Charco \\
\hline Charcos & Chinga & Chira & Chiva \\
\hline Chivo & Cholos & Chonta & Chompipe \\
\hline Chompipa & Chorizo & Chorro & Chumico \\
\hline Ciego & Ciruelas & Claro & Coco \\
\hline Cofradía & Conejo & Concha & Congo \\
\hline Coro & Coyote & Crema & Cruce \\
\hline Cuesta & Cueva & Culebra & Cápsulas \\
\hline Cusuca & Cusuco & Danta & Delicias \\
\hline Delirio & Divisa & Dicha & Duende \\
\hline Duendes & Duquesa & Encanto & Ensayo \\
\hline Eriza & Esperanza & Espuela & Estaca \\
\hline Eslabones & Estrella & Estanque & Faro \\
\hline Fierro & Flores & Fortuna & Frío \\
\hline Gallina & Gallo & Gardenia & Garza \\
\hline Garrobo & Gata & Gatas & Gavilanes \\
\hline Gavilana & Giganta & Gaviota & Gemelas \\
\hline Golondrinas & Gongolona & Gorda & Gordo \\
\hline Gorriones & Gradas & Grana & Grande \\
\hline Gringo & Gringos & Guacamaya & Guacamayo \\
\hline Guacas & Guácimo & Guape & Guapes \\
\hline Guatusa & Guayabo & Guayabos & Guineo \\
\hline Guinea & Guiones & Hacha & Hamaca \\
\hline Hermosa & Honda & Horcones & Hormiga \\
\hline Horno & Huerta & Huevos & Humo \\
\hline Huracanes & India & Indios & Indio \\
\hline Imágenes & Isla & Infierno & Inocentes \\
\hline Jícaro & Jirona & Jicote & Jobo \\
\hline Jocote & Juncos & Jobitos & Junta \\
\hline Juntas & Lagarta & Lagarto & Lágrimas \\
\hline Lajas & Lapa & Larga & Lechuga \\
\hline Lechuza & Leona & Letras & Limón \\
\hline Limones & Loba & Lisa & Llano \\
\hline Loma & Lomas & Lora & Loras \\
\hline Lucha & Loros & Machaca & Machaca \\
\hline Machete & Macho & Mango & Maquenca \\
\hline Maquenco & Maravilla & Martirio & Mata \\
\hline Mero & Mica & Mico & Melón \\
\hline Mesas & Micos & Miga & Mina \\
\hline Minas & Mocho & Mono & Monos \\
\hline Montaña & Mansión & Mora & Moras \\
\hline Morenas & Morros & Mosca & Mudos \\
\hline Marimba & Muerto & Muertos & Mula \\
\hline
\end{tabular}




\begin{tabular}{|c|c|c|c|}
\hline Mulas & Muñeco & Muñecos & Murciélago \\
\hline Nance & Naranjo & Naranjos & Nariz \\
\hline Negro & Negra & Nicho & Niñas \\
\hline Níspero & Norte & Nubes & Obispo \\
\hline Ojoche & Olivares & Ombligo & Oriente \\
\hline Oliveros & Orgullo & Oro & Ortiga \\
\hline Oscuro & Overo & Pájaros & \\
\hline Palmas & Panteón & Palma & Pampas \\
\hline Panales & Papayo & Papaturro & Paraíso \\
\hline Pargos & Patios & Patos & Pava \\
\hline Pavas & Peje & Penca & Pendeja \\
\hline Perico & Perla & Perra & Peña \\
\hline Perro & Perros & Piches & Piedra \\
\hline Piedras & Pila & Pilas & Pensamiento \\
\hline Picuda & Piragua & Planes & Pita \\
\hline Pizote & Placeres & Planes & Plata \\
\hline Playa & Polos & Pleito & Pochote \\
\hline Pozas & Pozo & Presa & Prieta \\
\hline Pretil & Pulga & Queque & Quesos \\
\hline Prodigio & Puercos & Primero & Puente \\
\hline Raíces & Raíz & Rama & Ramos \\
\hline Rico & Raspa & Rayo & Real \\
\hline Redondo & Rey & Rica & Recreo \\
\hline Róbalo & Roble & Rodeo & Rosas \\
\hline Rueda & Reforma & Reina & Rescate \\
\hline Sábalo & Sahíno & Salto & Sanguijuela \\
\hline Sardina & Sardino & Sardo & Sordo \\
\hline Seca & Seco & Serena & Sereno \\
\hline Silencio & Sucio & Soga & Susto \\
\hline Tabaco & Tallo & Tajo & Taranta \\
\hline Tejona & Tempate & Terciopelo & Toneles \\
\hline Tibio & Tiburón & Tigra & Torno \\
\hline Toro & Tieso & Toros & Tortuga \\
\hline Tractor & Trapiche & Tumbas & Triunfo \\
\hline Troja & Troncos & Turco & Tuna \\
\hline Trancas & Unión & Urraca & Vaca \\
\hline Venado & Venados & Ventanas & Verde \\
\hline Vieja & Viejo & Vigía & Virgen \\
\hline Viuda & Vainilla & Vegas & Vueltas \\
\hline Veraneras & Vivero & Volcán & Yegua \\
\hline Yuca & Yugo & Zacate & Zapote \\
\hline Zompopa & Zopilota & Zopilote & Zorra \\
\hline Zoncho & Yurros & Zanja & Zorra \\
\hline
\end{tabular}


GARITA: Morfemas derivativos y flexivos en la formación toponímica de la provincia de Guanacaste... 151

\subsubsection{Raíces atadas con morfemas derivativos y flexivos}

\begin{tabular}{|c|c|c|c|}
\hline Asientill - o & Azufral - es & Corozalit - o & Manzanal - es \\
\hline Caballit - o & Cerrit - o & Cerrit - o-s & Polvazal - es \\
\hline Ramad-a & Rancherí - a & Casit - a - s & Ceibit - a \\
\hline Concavit - $\mathrm{a}-\mathrm{s}$ & Corocit - o-s & Coyolarcit - o-s & Huaquit - a - s \\
\hline Huaquit - o-s & Iguanit - a - s & Lajit - a - s & Lomit - a - s \\
\hline Naranjit - o - s & Morrit - o - s & Ranchit - o-s & Nacascolit - o \\
\hline Sabalit - o & Negrit - o-s & Salinit - $\mathrm{a}-\mathrm{s}$ & Papelit - o \\
\hline Talolinguit - a & Pocit - a - s & Pocit - o & Pocit - o-s \\
\hline Yeg, it - a - s & Zanjit - a & Pilit - a - s & Ranit - a \\
\hline Saltit - o & Sequit - o & Tempisquit - o & Tronadorcit - a \\
\hline Orosilit - o & Clavelin - $\mathrm{a}-\mathrm{s}$ & Asientill - o & Bolill - o-s \\
\hline Arenill - a & Boquet - e & Brojud - o & Conventill - o - s \\
\hline Cornizuel - o & Corralill - o & Esterill - $\mathrm{a}-\mathrm{s}$ & Guanacastill - o \\
\hline Hatill - o & Camastr - o & Hoguerill - a & Honill - a - s \\
\hline Islet - a & Lagartill - o & Platanill - o & Leoncill - o \\
\hline Plazuel - a & Playit - a - s & Portill - o & Pajarit - o \\
\hline Potrrill - o-s & Manzanill - o & Montecill - o & Piñuel - a \\
\hline Zapatill - o & Coralill - o & Crucill - a - s & Jocotill - o \\
\hline Molinill - o & Palizad - a & Picud - a & Picud - a-s \\
\hline Quebrach - o & Sabanill - a & Granadill - a & Lagun - a \\
\hline Angostur - a & Calentur - a & Desprendimient - o & Florid - a \\
\hline Contenter - a & Henchider - o & Pelon - a & Pelon - a - s \\
\hline Pescader - o & Pintur - a & Virador - es & Goter - a \\
\hline Arenos - a & Barros - a & Calos - a & Colorad - o \\
\hline Bullicios - a & Espavelos - a & Gusanos - a & Lajos - a \\
\hline Pedregos - a & Salin - as & Salinit - a - s & Sainos - a \\
\hline Salin - a & Cenizos - a & Aserrader - o & Frances - a \\
\hline Bebeder - o & Botader - o & Caler - a & Carboner - a \\
\hline Coyoler - a & Chiquer - o & Choricer - a & Coyoter - a \\
\hline Cementeri - o & Embarcader - o & Hormiguer - o & Linder - o \\
\hline Lagarter - o & Poneder - o & Hueser - a & Prader - a \\
\hline Moleder - o & Queser - a & Cabecer - a - s & Despeñadero \\
\hline Divisader - o & Lajer - o & Resbalader - o & Raspader - o \\
\hline Sombrer - o & Potrer - o & Terrer - o & Terrer - o - s \\
\hline Tiester - o & Tranquer - a & Tucer - a & Callejon - es \\
\hline Lloron - a & Dormilon - a & Esteron - es & Lajon - es \\
\hline Playon - es & Pajaron - es & Peñasc - o & Pilon - a - s \\
\hline Zanjon - es & Boqueron - es & Charcon - es & Tronador - a \\
\hline Rancherí - a & Cajet - a & Calcet - a & Calet - a \\
\hline $\begin{array}{l}\text { Islet - a } \\
\text { Cociner - o }\end{array}$ & Pilet - a & Boquet - e & Carret - a \\
\hline
\end{tabular}




\subsubsection{Raíces atadas formadoras de adjetivos derivados de participios}

\begin{tabular}{|c|c|c|c|}
\hline Arrepentid - o - s & Desjarretado - d - o & Rajada - d - o & Escondi - $\mathrm{d}-\mathrm{a}$ \\
\hline Escondi - $\mathrm{d}-\mathrm{o}$ & Reventa - d - o & Rompi - d - o & Atravesa - d-o \\
\hline Raspa - d - o & Ahog -(a) - d - o & Ahog -(a)-d-o-s & Azufr- a - d-a \\
\hline Azufr $-a-d-o$ & Muer - $t$ - o & Muer - $t-o-s$ & $\operatorname{Pel}(a)-d-a$ \\
\hline $\operatorname{Pel}(a)-d-o$ & $\operatorname{Pel}(a)-d-o-s$ & Color(a) - d - o & \\
\hline
\end{tabular}

\section{Análisis de los morfemas que entran en la formación de topónimos}

Los topónimos de la provincia de Guanacaste presentan gran variedad de morfemas. Aparecen tanto derivativos como flexivos. Los morfemas derivativos más frecuentes son los sufijos: 326.

No se presentan topónimos con infijos como formantes, lo que corrobora una característica del léxico del español: la casi ausencia del fenómeno de infijación. Por otra parte, es muy escasa la presencia de topónimos con prefijos; en total, 14.

De los topónimos sufijados, ocupan un primer lugar los abundanciales; en total, 105 con un $32,2 \%$. Le siguen los diminutivos, 85 , para un $26 \%$. Con morfema despectivo aparecen 36 que corresponden a un $11 \%$. Los morfemas que significan lugar, instalación o vinculación con algo son 34 y corresponden a un 10,4\%. Un grupo de 17 morfemas con significado de acción, efecto o acción-efecto presentan un 5,2\%; 19 morfemas se refieren a cualidades de los topónimos y constituyen un 5,8\%. Finalmente, en menor cantidad, tenemos 4 morfemas que significan agente, dos con significado de gentilicio, tres con morfema que intensifica la cualidad y uno con significado colectivo, que en total corresponden a un 3,06\%.

El morfema abundancial presenta tres alomorfos, a saber, -al, -ar, y -ada. Los alomorfos -al y -ar son mayoría y están condicionados fonéticamente, es decir, el alomorfo -ar aparece cuando el topónimo tiene en su raíz el fonema / 1/. Por ejemplo en balsar, brasilar, colmenar, coyolar; sin embargo hay dos topónimos con raíz bals- en los que aparecen indistintamente los alomorfos-al y -ar, lo que implica una vacilación en el uso de ambos. Es menos frecuente el alomorfo -ada, pues, de los 105 abundanciales sólo hay cinco en -ada, lo cual es poco significativo.

Los morfemas diminutivos, en total 82, son más variables; presentan 6 alomorfos.

Los topónimos con morfema -it, en algunos casos, corresponden a un topónimo que está cerca de otro topónimo con el mismo nombre; el diminutivo indica que el otro accidente o poblado es más pequeño. Por ejemplo Tempisque y Tempisquito; Alajuela (Provincia), Alajuelita (Poblado); Brasil (Río), Brasilito (Río); Caimital, Caimitalito; Aguacate (Río), Aguacatico (Quebrada); Nacascolo (Bahía), Nacascolito (Playa).

El alomorfo -it es mayoría; aparecen, además, dos topónimos con el alomorfo -cit: Coyolarcita y Tronadorcita; dos topónimos con el alomorfo -ecit: Finca Florecita y Finca Lucecita; dos topónimos con el alomorfo -in: Quebrada Molino y Río Clavelinas. Dos alomorfos -uel, Piñuela que corresponde al nombre de un cerro y una quebrada. Dentro de estos morfemas diminutivos se destaca, también, un morfema que expresa una disminución de tamaño, sin llegar a ser el mínimo. Es un significado intermedio entre el grande y el pequeño; me refiero al sufijo -et, que 
podría tomarse como un morfema y no como un alomorfo de diminutivo; no obstante, por tratarse de tamaño, prefiero ubicarlo aquí; por ejemplo quebrada Piletas o cerro Boquete.

El morfema con significado despectivo presenta siete alomorfos. Hay un predominio del alomorfo -ill. Aparecen otros alomorfos como -cill en isla Leoncillo, -ecill en laguna Montecillo, -iz- en cerro Palizada, -cho en Cerro Quebracho, -un en finca Laguna y -stro en loma Camastro.

El siguiente grupo de topónimos tiene un morfema que expresa lugar, instalación o vinculación con algo. Es muy uniforme pues no tiene alomorfos. El único morfema es -er, sin embargo, los significados no son exactamentre iguales aunque sí bastante relacionados; tal es el caso del poblado Embarcadero, lugar donde se embarca; el río Campero, perteneciente o vinculado con el campo; playa Cementerio, poblado Lindero, instalación de un cementerio o un límite respectivamente.

Para el morfema con significado de aumentativo aparecen únicamente dos alomorfos: -on y -asc. Por supuesto, el alomorfo -on es mayoría y solamente un topónimo presenta el alomorfo -sco en Quebrada Peñasco.

Los morfemas bajo el significado de acción, efecto o acción-efecto son más variados en cuanto a sus alomorfos: -ur en un poblado Angostura; -mient en Desprendimiento, acción o efecto de desprender; -id en quebrada Florida, el efecto de florecer; -er en río Henchidero, acción-efecto de henchir; -on en cerro Pelón, acción-efecto de pelar; -dor en bahía Virador, acción-efecto de virar; -ción en cerro Coronación, acción-efecto de coronar.

El siguiente grupo de topónimos presenta un morfema que expresa cualidad física o abstracta. El alomorfo -os es el más común y se refiere a cualidades físicas. Con el alomorfo -in aparece el nombre de un poblado, un estero y una punta. El topónimo es Salinas, que tiene sal; también el nombre del río Salina. Las cualidades en el sentido abstracto tienen como alomorfos - dad en finca Felicidad y -anza en quebrada Esperanza.

En el corpus estudiado aparece, finalmente, un grupo pequeño de topónimos formados por un morfema con significado de agente. Los alomorfos son -dor en finca Chupador, finca Escarbador, poblado y río Tronadora. También hay un alomorfo -er, que forma el nombre Ranchería. En este mismo topónimo, el sufijo -í expresa colectividad.

Con significado de gentilicio están los sufijos -es en el nombre finca Francesa, -ol en finca Española y -er con significado de oficio en el topónimo finca Cocinero.

Hay tres nombres que presentan un mismo morfema -ud, cuyo significado consiste en intensificar la función básica de la raíz. Se trata de los topónimos Brojudo (cerro), Picuda (cerro y loma) y Picudas (fila).

Respecto de los morfemas derivativos tipo prefijos, como ya se explicó, son muy escasos. Se presenta el morfema derivativo -des en quebrada Deshechos, poblado Desjarretado, quebrada Desengaño y quebrada Desprendimiento. Otro morfema en- con significado dentro en los nombres poblado Embarcadero, río Enmedio y quebrada Enramada. El prefijo re- con significado de nuevamente se encuentra en los nombres loma Rempujo y finca Reforma y otro morfema re- con significado de superlativo "muy" aparece en el nombre finca Refundores que significa muy adentro de la montaña y cerros Rejoya cuyo significado es muy hueco o profundo. Finalmente, hay dos nombres con la preposición a como prefijo (a-) que expresa dirección: punta Abajo y cerro Atravesado. 
En cuanto a los morfemas flexivos, a pesar de que en el corpus son muy numerosos, al ser clases cerradas, no presentan gran variedad. Por ser sustantivos o adjetivos, sus morfemas flexivos son típicos: morfemas de género masculino o femenino y de número plural. Además, en los adjetivos provenientes de un participio verbal se presenta el morfema característico de participio - $\mathrm{d}$ y en dos casos - $t$.

Un aspecto interesante en cuanto al género es la arbitrariedad en la asignación de morfemas para masculino y para femenino. No hay ninguna regla posible de establecer, ni aún tomando en cuenta el género gramatical del nombre genérico pues no hay concordancia en muchos casos. Así se encuentra quebrada Chiquito, quebrada Cusuca y laguna Cusuco, poblado Florida, cerro Picuda, cerro Picudo. La razón es que se respeta el morfema de género de los componentes del ambiente que han sido tomados para darle nombre al topónimo y que, a su vez, presentan gran variedad en sus alomorfos. Respecto del morfema número, los alomorfos son -s o -es.

\section{Conclusiones}

Los topónimos de la provincia de Guanacaste presentan, en su formación, morfemas flexivos y derivativos.

Los morfemas flexivos corresponden a las marcas de género masculino o femenino. No se presenta regularidad en cuanto a estos morfemas debido a que la mayoría de las veces el poblado o accidente geográfico toma su nombre de algún componente del ambiente que puede ser, a su vez, masculino o femenino, por lo que, no se da concordancia o relación alguna. Los morfemas más comunes son -o, -e para masculino y femenino. Además el femenino presenta los morfemas -a, -esa, -ina. El número es más regular en cuanto a morfemas. Los que aparecen en este corpus son -s y -es.

Los morfemas derivativos, por su parte, presentan más variedad. Constituyen mayoría los abundanciales seguidos de los diminutivos, los despectivos, los de lugar, los de acciónefecto, los aumentativos y los que se refieren a cualidades de los topónimos. Con un porcentaje muy bajo aparecen morfemas con significado de agente, gentilicio y oficio, e intensificadores de cualidad.

En cuanto a la diversidad de alomorfos para cada morfema, el que presenta más variedad es el morfema con significado despectivo, seguido por los morfemas con significado diminutivo, de acción-efecto y de cualidad.

El morfema abundancial, a pesar de ser mayor en número, sólo presenta tres alomorfos y el aumentativo, dos.

Finalmente, hay dos morfemas bastante uniformes: uno que significa lugar, con el alomorfo -er y otro que intensifica la cualidad con el alomorfo -ud. 
GARITA: Morfemas derivativos y flexivos en la formación toponímica de la provincia de Guanacaste... 155

\section{Bibliografía}

Fuentes, Juan Luis. 1985. Gramática moderna de la Lengua Española. Madrid: Edime. Organización Gráfica S.A.

Hocket, Ch. 1971. Curso de Lingüística Moderna. Tercera Edición Argentina: Editorial Universitaria.

Mathews, P.H. 1980. Morfología. Madrid: Editorial Paraninfo S.A.

Real Academia Española. 1986. Esbozo de una nueva Gramática de la lengua española. Madrid: Editorial Espasacalpe S.A. 